REVISTA NORMAS, N. ${ }^{\circ}$ 4, 2014, PP. 93-116 (ISSN: 2174-7245)

(RECIBIDO: 01-06-2014. EvaluADO: 15-06-2014. ACEPTADO: 15-07-2014)

https://doi.org/10.7203/Normas.4.4689

\title{
CORTESÍA Y LENGUAS DE ESPECIALIDAD ENTRE LO LOCAL Y LO GLOBAL: EL CASO DE LAS RESEÑAS DE HOTELES EN ESPAÑOL E INGLÉS
}

\author{
POLITENESS AND LSP BETWEEN THE LOCAL AND THE GLOBAL: \\ HOTEL REVIEWS IN SPANISH AND ENGLISH
}

Sara PICCIONI

Università G. D’Annunzio di Chieti-Pescara

\section{RESUMEN:}

El presente trabajo analiza el género de la reseña de hoteles en español y en inglés para explorar la evolución de este género a raíz de su internacionalización. El análisis basado en corpus se realiza a través de una comparación monolingüe y bilingüe contrastiva, observando la distribución de estrategias de atenuación e intensificación (Briz, 2001; Albelda, 2007) de los adjetivos con carga semántica negativa en tres tipos de reseñas: reseñas en español publicadas en plataformas nacionales españolas, reseñas en español publicadas en plataformas internacionales, y reseñas en inglés publicadas en plataformas internacionales. Los resultados cuantitativos demuestran que la difusión global de estos textos promueve la fidelidad al género internacional más que a la tradición discursiva de la lengua y cultura nacionales, al mismo tiempo que limita los rasgos lingüísticos típicos de lo coloquial, dando paso a una nueva forma de comunicación especializada.

PALABRAS CLAVE: reseñas de hoteles, turismo 2.0, atenuación, intensificación, lingüística de corpus.

\section{ABSTRACT:}

This paper analyses hotel reviews in Spanish and English to explore the evolution of this genre as a consequence of its availability on the globalised tourism market. A corpus-based comparison (monolingual and bilingual contrastive) is carried out of hedging and intensifying strategies (Briz, 2001; Albelda, 2007) used to modify semantically negative adjectives. The distribution of these strategies is observed across 3 corpora comprising respectively: reviews in Spanish published on Spanish platforms; reviews in Spanish published on international platforms; reviews in English published on international platforms. Quantitative results show that global circulation of this genre promotes convergence towards the international genre rather than preservation of the national discourse tradition; such convergence discourages the use of linguistic traits typical of informal settings, giving rise to a new form of specialised communication.

KEYWORD: hotel reviews, tourism 2.0, hedges, internsifiers, corpus linguistics. 


\section{INTRODUCCIÓN}

El presente trabajo se centra en el impacto que la globalización del mercado del turismo en Internet y la participación directa del viajero en la difusión de información turística producen en las características de los géneros discursivos de este ámbito. Las posibilidades de interacción brindadas por la web 2.0 están provocando cambios profundos en las formas de la comunicación turística: entre estos cabe señalar el protagonismo adquirido por la comunicación entre viajeros para la difusión de contenidos y la estandarización a nivel global de las formas de comunicación a través de la adopción de moldes discursivos compartidos dentro de una comunidad internacional. Este segundo aspecto resulta de particular interés, ya que se puede suponer que la necesidad de estandarización favorece la adopción y difusión de géneros discursivos estables, caracterizados por rasgos funcionales y formales compartidos, que convierten la comunicación entre pares en una forma de comunicación especializada.

El estudio que aquí se presenta parte de esta hipótesis, y se propone verificarla con un análisis basado en corpus de opiniones sobre hoteles, un género emergente de la comunicación turística actual en el que el viajero comparte con los demás miembros de la comunidad virtual sus opiniones y consejos acerca de un establecimiento hotelero específico. Con el propósito de explorar la evolución de este género a raíz de la internacionalización de su uso, el análisis se realiza a través de un doble tipo de comparación, monolingüe y bilingüe contrastivo, cotejando tres tipos de opiniones: opiniones en español publicadas en plataformas nacionales españolas, opiniones en español publicadas en plataformas multilingües internacionales, y opiniones en inglés publicadas en plataformas internacionales. De esta forma se pretende, por un lado, observar las características propias de las opiniones en español y, por otro, verificar si la publicación en plataformas internacionales determina una convergencia hacia las convenciones discursivas del modelo dominante inglés.

El análisis se realizará a partir de la observación del uso de los adjetivos en los tres corpus, ya que esta categoría morfosintáctica realiza la importante tarea de vehicular descripciones y valoraciones, tarea vinculada a las funciones prototípicas del género analizado. En particular, a partir de listados de frecuencia y del análisis de los clusters (patrones léxicos recurrentes) asociados a los adjetivos con carga semántica negativa, se describirán las estrategias de atenuación e intensificación de los adjetivos, observando su distribución en los tres corpus. Esta distribución se utilizará para caracterizar el registro de los textos de cada uno de los corpus, poniéndolo en relación con su nivel de especialidad.

En los apartados que siguen, se describen los rasgos comunicativos del género analizado (apartado 2) y se sientan las bases teóricas del estudio, vinculando el nivel de especialización de los textos a los rasgos situacionales y comunicativos de la comunicación formal (cfr. Briz, 2010; apartado 3.1) y a los mecanismos de atenuación e intensificación (Briz, 2001, Albelda, 2007; apartado 3.2). En el apartado 4 se describen los mecanismos de 
atenuación e intensificación observados, ilustrando su distribución cuantitativa en los tres corpus. Las conclusiones (en el apartado 5) reflexionan sobre las implicaciones de los resultados obtenidos, que demuestran que la difusión global de estos textos promueve la fidelidad al género internacional más que a la tradición discursiva de la lengua y cultura nacionales, al mismo tiempo que limita los rasgos lingüísticos típicos de lo coloquial, dando paso a una nueva forma de comunicación especializada.

\section{RESEÑAS DE HOTELES: PRESTIGIO Y CORTESÍA EN LA COMUNICACIÓN GLOBAL}

En años recientes, uno de los factores que más cambios ha producido en el mundo del turismo es la disponibilidad de las tecnologías de la llamada «web 2.0», que han favorecido una más intensa interacción entre proveedores de servicios turísticos y clientes potenciales y reales, otorgando además a estos un nuevo protagonismo en la comunicación turística. Las nuevas tecnologías han modificado de forma profunda el proceso de toma de decisiones en la elección de metas y establecimientos turísticos, a través de una nueva forma de boca a boca, virtual y global, que demuestra que quien se prepara para un viaje confía en la opinión de los miembros de la comunidad virtual para decidir adónde ir, qué visitar, dónde alojarse, dónde comer, etc. El fenómeno ha adquirido una gran relevancia comercial, ya que se ha comprobado que las opiniones de otros viajeros repercuten de forma determinante en la decisión de los clientes potenciales de hacer una reserva o comprar un producto ( $c f r$. Vermeulen y Seegers, 2009).

Por su capacidad de influir en la conducta de los consumidores, estos textos se han convertido, indirectamente, en una nueva forma de promoción de los establecimientos turísticos; sin embargo, desde un punto de vista comunicativo y discursivo, presentan peculiaridades propias que los distinguen del discurso de la promoción turística tradicional. Estas peculiaridades se pueden relacionar a dos aspectos principales: primero, la identidad e intenciones de los autores; segundo, la dimensión internacional de la producción y la recepción de los textos.

Por lo que atañe al primer aspecto, los autores de opiniones son personas que han viajado por placer más que los viajeros que no escriben reseñas; frente a estos, están mayormente implicados en la planificación del viaje y, para ello, usan con más frecuencia la red y, en particular, redes sociales y chats (Yoo, Gretzel y Purifoy, 2007). Los estudios que han investigado las motivaciones que mueven los consumidores a convertirse en autores de reseñas (cfr. Hennig-Thurau et al., 2004; Ho y Dempsey, 2010; Mackiewicz, 2010; Tian, 2013) han evidenciado una combinación de motivaciones altruistas y egoístas, que se pueden resumir en:

- Preocupación por los demás, intención altruista de ayudar a otros consumidores (Recomendable $100 \%$, No cometáis el mismo error que nosotros); 
- Voluntad de interactuar socialmente participando en la comunidad virtual e identificándose en ella;

-Reconocimiento y promoción personal como viajero experto cuya opinión merece ser tenida en cuenta (He ido varias veces a este hotel porque me gusta mucho en todas sus facetas; Viajo mucho a la capital pucelana y tengo que decir que siempre paro en este gran hotel por los motivos que luego explicare);

-Ejercicio del poder colectivo de los consumidores sobre los establecimientos.

Estos datos proporcionan elementos importantes para caracterizar las funciones de las opiniones y las intenciones de sus autores: se trata de textos escritos fuera del marco de la comunicación profesional, cuya función primaria no es promover un producto, sino una opinión. Como en los textos promocionales tradicionales, el emisor desea influir en la conducta de quien lee; sin embargo, en este caso ese influjo se busca como forma de reconocimiento de la validez o prestigio de las opiniones personales expresadas. De ahí la naturaleza argumentativa de estos textos, en los que quien escribe se preocupa por construir y preservar su imagen y reputación, buscando el acuerdo del interlocutor. La relevancia que la negociación de las imágenes de emisor y destinatario tiene en la caracterización de este género motiva la decisión de centrar este estudio en aspectos esencialmente pragmáticos, para observar cómo esas imágenes se construyen y defienden a través de estrategias de gestión de la cortesía.

El segundo aspecto sobre el que se quiere llamar la atención es la dimensión internacional que las opiniones adquieren gracias a la difusión en plataformas globales que imponen la estandarización de las formas de comunicación, a menudo a través de la promoción de un modelo dominante. En este contexto, es interesante ver cómo las distintas tradiciones discursivas adoptan y/o adaptan ese modelo dominante y cómo estas dinámicas influyen en la definición de un marco formal reconocible y estable. Como ya se ha mencionado, en este trabajo se avanza la hipótesis de que la adopción por parte de la comunidad nacional de viajeros españoles de un género discursivo globalizado contribuye a la creación de un molde formal estable y compartido por los integrantes de esta comunidad discursiva, convirtiéndose por lo tanto en una forma de comunicación especializada: de ahí la decisión de denominar los textos analizados reseñas, y no opiniones.

\section{INTERSECCIÓN ENTRE DISCURSO FORMAL Y DISCURSO ESPECIALIZADO}

Para caracterizar las reseñas de hoteles como un género especializado se propone observar su nivel de formalidad. Si bien Briz (2010: 127) subraya que «se puede hablar coloquialmente el lenguaje de especialidad», las situaciones comunicativas en las que suele producirse la comunicación especializada se pueden asociar con frecuencia a los rasgos que 
el autor llama «de formalidad», acercando sus formas expresivas a la llamada «constelación comunicativa formal».

Según el autor (Briz, 2010: 128), el prototipo de lo formal se caracterizaría por la falta de:

- relación social o funcional [de igualdad] entre los interlocutores;

- relación vivencial de proximidad entre estos;

- marco interaccional familiar;

- cotidianidad temática de la interacción;

- planificación sobre la marcha;

- fin interpersonal;

- tono informal.

En el caso de las reseñas hoteleras, algunas de estas dimensiones pueden variar de forma significativa dependiendo de las intenciones de los emisores. Los ejemplos que siguen lo demuestran de forma muy clara:

(1) justo lo que esperabamos...el entorno genial......facil de llegar....silencioso...atractivo.... nada mas llegar ya te llama la atencion......la suite 4 estaciones esta muy my muy bien....no le falta detaye...la tele rotatoria es la caña y el hidromasaje......brutal.........y si que se llena en menos de 4 horas......comprobado el sabado por la noche....unos a las 8 y otro a las 11:30 y los dos ardiendo....lo dejamos un rato ke se enfriase xq madre mia......detayazo la botella de vino espumoso muy ricoo....esta muy bien insonorizado pero esque en si el sitio es tranquilo....y ademas me parecio ideal para los mas pequeños ....tiene 2 o 3 parques apañaikos....bueno esta es mi opinion....a quien no le guste pliin!!!! [ES_ES].

(2) Es un hotel con una buena relación calidad precio. Está limpio y es moderno. Está algo apartado del centro, unos 25 minutos a pie, pero tiene parada de autobús cerca. La habitación es espaciosa para dos personas, pero si se pide una cama supletoria, se queda algo justa de espacio. El baño es amplio y bien equipado. Ofrecen un desayuno correcto. En agosto cierra la cocina, no se puede cenar ni comer salvo bocadillos y similares. El trato es muy bueno. Los propietarios ofrecen todo tipo de información y son bastante agradables al trato [ES_INT].

En (1), quien escribe establece una relación funcional de igualdad con los demás viajeros del foro, poniendo énfasis en una supuesta proximidad vivencial con estos. El foro se interpreta como un marco interaccional familiar, en el cual se comparten contenidos cotidianos no especializados. Los puntos suspensivos y la sintaxis fragmentaria indican el limitado nivel de planificación; la comunicación, aun no dejando de ser informativa, hace hincapié en el fin interpersonal al utilizar un tono marcadamente informal, como sugieren (entre otras cosas) el léxico elegido (la tele rotatoria es la caña y el 
hidromasaje......brutal.........), la ortografía (lo dejamos un rato ke se enfriase xq madre mia......detayazo la botella de vino), el constante uso del énfasis (muy my muy bien; muy ricoo). En (2), en cambio, el autor de la reseña resalta implícitamente la autoridad que su experiencia de viajero experto le otorga, interponiendo una marcada distancia entre sí y el interlocutor y lo dicho, como demuestran las formas impersonales utilizadas (no se puede cenar ni comer; Los propietarios ofrecen todo tipo de información); el foro se interpreta como el marco para una interacción formal, bien planificada y caracterizada por una finalidad marcadamente transaccional. Es evidente que (1) se coloca en la «constelación comunicativa coloquial», mientras que (2), aun manteniendo rasgos coloquiales, se acerca de manera bastante clara a la «constelación formal».

Ahora bien, finalidad transaccional, contenidos especializados, distancia vivencial entre los interlocutores, marco interaccional no familiar son, además de rasgos de formalidad, características que tienden a asociarse con la comunicación especializada. De hecho, no es difícil interpretar (2) como un texto con mayor nivel de especialización frente a (1).

\subsection{Atenuación e intensificación}

Para comparar el nivel de especialización/formalidad de reseñas nacionales e internacionales se tomarán en consideración los rasgos lingüísticos asociados a las estrategias de atenuación e intensificación. Se trata de dos conceptos que han ido adquiriendo definiciones pragmáticas en relación al grado de compromiso del hablante con lo dicho, convirtiéndose también en elementos capaces de caracterizar el eje de lo coloquial y lo formal que nos interesa.

Según Briz (2003: 19):

la atenuación, como categoría pragmalingüística, es una operación lingüística estratégica de minimización de lo dicho y del punto de vista, así pues, vinculada a la actividad argumentativa y de negociación del acuerdo, que es el fin último de toda conversación. Quitar relieve, mitigar, suavizar, restar fuerza ilocutiva, reparar, esconder la verdadera intención son valores más concretos unidos al empleo del atenuante [...]

Se trataría, por lo tanto, de un recurso «que busca la aceptación del oyente» (Briz, 2003: 17), modulando el impacto del contenido proposicional (lo dicho) y/o la fuerza ilocutiva del acto de habla (el decir). Con la atenuación de lo dicho «se minimiza la cantidad o se expresa un elemento de la proposición de forma vaga o imprecisa», a través de recursos morfológicos (por ejemplo, Solo les robaré unos minutitos para explicarles mi oferta) o léxicos (con eufemismos como en Las empresas económicamente débiles no podrán afrontar la subida de impuestos) (Albelda, 2010: 58). La atenuación de la fuerza ilocutiva hace uso de distintos mecanismos lingüísticos según el tipo de actos atenuados: solo por poner algún ejemplo, se citan aquí el uso del condicional y del imperfecto para 
atenuar los actos directivos (por ejemplo, quería preguntarte si podrías traducirme un par de cosas del japonés) o los adverbios modalizadores para mitigar la fuerza de los asertivos (Quizás se lo haya sugerido así Fidel Castro durante su visita a Cuba) (ibid.: 51-52).

Frente a la atenuación, la intensificación se perfila como una estrategia evaluativa de maximización del compromiso del hablante con lo dicho y/o el acto del decir. Dentro de la intensificación cabe una amplia variedad de recursos lingüísticos, que en opinión de Briz (2001: 127-129) pueden tener dos funciones distintas: una función semántico-pragmática de realce del contenido proposicional a través de intensificadores de la cantidad y de la cualidad y una función puramente pragmática que atañe a «la fuerza ilocutiva de un acto o la presencia de los participantes en la enunciación»a través de los mecanismos de la modalidad (Briz, 2001: 129). Con ambos tipos de realces, el hablante refuerza su presencia y la de sus sentimientos, actitud, juicio, etc., al mismo tiempo que implica (in)directamente al interlocutor en lo dicho. A continuación, se proponen solo algunos ejemplos de los muchos recursos de intensificación identificados en literatura:

(3) Sabido y archisabido es que la misión del docente universitario comprende tres básicas actividades: enseñar, investigar y formar.

(4) Bueno, espero que no me lluevan piedras por abrir este hilo, pero es que ya estoy hasta las cejas del tema

(5) ...con lo mucho que está comiendo, pues agradece que no sea carnívora jajaja

(6) Y si, el año ke viene a Montmelo vamos fijo (este año fuimos a Jerez y el bolsillo no da para tanto).

(7) claro como no me va a gustar que te vallas si no te soporto osa.

Los ejemplos en (3), (4) y (5) utilizan intensificadores semánticos-pragmáticos correspondientes a recursos morfológicos (archisabido), léxicos (estoy hasta las cejas) y sintácticos (con lo mucho que); mientras que (6) y (7) refuerzan la modalidad epistémica a través de los adverbios fijo y claro.

Atenuación e intensificación se pueden asociar a rasgos situacionales típicos de registros formales y coloquiales. Albelda (2010: 59), proponiendo una síntesis de trabajos anteriores (Briz, 2004; Haverkate, 2004; Albelda, 2008; Bravo, 2008), identifica la atenuación con marcos situacionales de tipo transaccional, en los cuales median distancia vivencial (desconocimiento) y desigualdad funcional/social entre los participantes. De esta forma, la atenuación quedaría vinculada a registros de la «constelación formal». La intensificación, en cambio, tiende a manifestarse en marcos situacionales típicos de lo coloquial, en los cuales (tal como apunta Hidalgo, 2011: 275):

los hablantes comparten suficientes elementos lingüísticos y extralingüísticos, desde una relación vivencial de proximidad, hasta una temática conversacional no especializada, pasando, muy probablemente, por una relación social y/o funcional de igualdad y un marco de interacción cotidiano. 
El fin transaccional y una tendencia a eludir temas familiares y cotidianos en favor de temas especializados permiten identificar la atenuación como un mecanismo propio de la comunicación especializada. Por otra parte, numerosos son los análisis que se han centrado en los recursos mitigantes utilizados, sobre todo en la comunicación académica, para lograr la aceptación por parte del interlocutor y para modular el compromiso de quien habla/escribe con lo dicho ( $c f r$. Hyland, 1998; Mendiluce y Hernández, 2005; Oliver del Olmo, 2006; Morales, Cassany y González-Peña, 2007).

En los apartados que siguen se propone un análisis de los mecanismos de atenuación e intensificación asociados al uso de adjetivos en un corpus de reseñas hoteleras al fin de identificar el nivel de formalidad y, a través de él, el nivel de especialización de los textos. La decisión de centrar el análisis en el uso de los adjetivos se debe a la carga valorativa que intrínsecamente esta categoría morfosintáctica posee y que, en el caso del género discursivo en consideración, puede tener una múltiple función expresiva, persuasiva e informativa. Se considera que el uso de atenuantes e intensificadores en relación a los adjetivos puede ser revelador de la función de cada uso, convirtiéndose en un potencial indicador del nivel de especialización de los textos.

\section{ATENUACIÓN E INTENSIFICACIÓN DE LOS ADJETIVOS EN RESEÑAS HOTELERAS}

\subsection{Corpus}

El corpus que se utiliza para el análisis se compone de los tres subcorpus siguientes:

- ES_ES: reseñas hoteleras en español publicadas en plataformas nacionales españolas;

- ES_INT: reseñas hoteleras en español publicadas en plataformas internacionales;

- ING_INT: reseñas hoteleras en inglés publicadas por usuarios de Reino Unido en plataformas internacionales.

La identificación y selección de las plataformas nacionales para la recopilación de ES_ES ha tenido en cuenta los siguientes criterios:

- que se tratara de plataformas disponibles solo en español u otra lengua peninsular;

- que la empresa responsable de la plataforma web fuera una empresa registrada en España no vinculada a grupos internacionales.

Siguiendo estos criterios, se seleccionaron las siguientes webs: http://www.hoteles.net/; http://www.muchoviaje.com/; http://www.hotelforo.com. 
Las reseñas en ES_INT y ING_INT se recopilaron a partir de plataformas internacionales multilingües en sus declinaciones nacionales ( $\underline{w w w . t r i p a d v i s o r} . e s \quad y$ www.tripadvisor.co.uk, www.booking.com, www.trivago.es y www.trivago.co.uk). La decisión de seleccionar únicamente reseñas publicadas por usuarios de Reino Unido para la recopilación de ING_INT se debe a la voluntad de restringir el corpus a hablantes nativos o casi nativos de una misma macrovariedad de la lengua inglesa; somos conscientes, sin embargo, que el hecho de que un usuario indique Reino Unido como país de procedencia/residencia no garantiza en principio la homogeneidad requerida.

La tabla 1 ilustra el número de reseñas, de palabras (token) que componen cada subcorpus y una indicación de la longitud media de las reseñas en cada uno de ellos.

\begin{tabular}{|l|c|c|c|}
\hline & ES_ES & ES_INT & ING_INT \\
\hline Número de reseñas & 3728 & 1676 & 1552 \\
\hline Número de token & 179676 & 178582 & 179134 \\
\hline Longitud media reseñas & 48.2 & 106.55 & 115.42 \\
\hline
\end{tabular}

Tabla 1. Número de reseñas y de token de cada subcorpus. Longitud media de las reseñas en cada corpus

Un primer dato de interés que proporciona la tabla 1 es el de la longitud media de las reseñas, que en las plataformas internacionales (tanto en español como en inglés) duplica la de las reseñas nacionales.

\subsection{Frecuencia, variedad léxica e intensificación de los adjetivos}

El paso preliminar del análisis ha consistido en la compilación del listado de frecuencia de los adjetivos contenidos en cada subcorpus, eliminando los adjetivos con frecuencia menor de 20 ( $<<20)$ (tabla 2).

\begin{tabular}{|l|l|l|l|l|l|}
\hline \multicolumn{2}{|c|}{ ES_ES } & \multicolumn{2}{c|}{ ES_INT } & \multicolumn{2}{c|}{ ING_INT } \\
\hline buen & completo & buen & céntrico & clean & safe \\
gran & familiar & limpio & inmejorable & friendly & spacious \\
amplio & ideal & perfecto & antiguo & good & tidy \\
limpio & encantador & amable & maravilloso & lovely & cheap \\
cómodo & alto & excelente & completo & nice & efficient \\
pequeño & espectacular & gran & malo & great & enjoyable \\
excelente & inmejorable & amplio & confortable & comfortable & fantastic \\
perfecto & caro & mejor & gratuito & excellent & fine \\
agradable & especial & estupendo & rural & helpful & huge \\
amable & gratuito & agradable & variado & quiet & polite \\
tranquilo & acondicionado & tranquilo & caro & close & real \\
bonito & impresionante & único & histórico & little & slight \\
acogedor & barato & recomendable & alejado & small & wonderful \\
moderno & variado & correcto & espectacular & free & amazing \\
\hline
\end{tabular}




\begin{tabular}{|c|c|c|c|c|c|}
\hline $\begin{array}{l}\text { nuevo } \\
\text { atento } \\
\text { recomendable } \\
\text { correcto } \\
\text { precioso } \\
\text { antiguo } \\
\text { estupendo } \\
\text { cuidado } \\
\text { único } \\
\text { céntrico } \\
\text { confortable } \\
\text { genial } \\
\text { mejor }\end{array}$ & $\begin{array}{l}\text { pleno } \\
\text { malo } \\
\text { exterior } \\
\text { justo } \\
\text { natural } \\
\text { rico } \\
\text { frio } \\
\text { necesario } \\
\text { rural } \\
\text { interior } \\
\text { peor } \\
\text { suficiente } \\
\text { peatonal }\end{array}$ & $\begin{array}{l}\text { bonito } \\
\text { genial } \\
\text { pequeño } \\
\text { atento } \\
\text { precioso } \\
\text { acogedor } \\
\text { ideal } \\
\text { moderno }\end{array}$ & $\begin{array}{l}\text { comercial } \\
\text { necesario } \\
\text { encantado } \\
\text { justo } \\
\text { imposible } \\
\text { peor } \\
\text { fenomenal }\end{array}$ & $\begin{array}{l}\text { pleasant } \\
\text { hot } \\
\text { modern } \\
\text { warm } \\
\text { best } \\
\text { convenient } \\
\text { full } \\
\text { reasonable } \\
\text { sure } \\
\text { welcoming } \\
\text { busy } \\
\text { cold } \\
\text { delicious } \\
\text { old } \\
\text { positive } \\
\text { short }\end{array}$ & $\begin{array}{l}\text { bad } \\
\text { better } \\
\text { big } \\
\text { central } \\
\text { decent } \\
\text { expensive } \\
\text { historic } \\
\text { larger } \\
\text { noisy } \\
\text { quick } \\
\text { sufficient } \\
\text { superb } \\
\text { uncomfortable } \\
\text { wet }\end{array}$ \\
\hline
\end{tabular}

Tabla 2. Listado de frecuencia de los adjetivos de cada subcorpus $(\mathrm{f}<20)$

A partir del listado de frecuencia, se han obtenido el número total de adjetivos, su frecuencia por 1.000 palabras en cada corpus y el type/token ratio como medida de variedad léxica específica de los adjetivos (Tabla 3).

\begin{tabular}{|l|c|c|c|}
\hline & ES_ES & ES_INT & ING_INT \\
\hline N. $^{\text {o de adjetivos }}$ & 9444 & 9310 & 9258 \\
\hline Frecuencia/1 000 palabras $^{\text {Type/token ratio adj. }}$ & 52.56 & 52.13 & 51.66 \\
\hline
\end{tabular}

Tabla 3. Frecuencia y variedad léxica de los adjetivos

A continuación, en cada listado de frecuencia se han seleccionado los «adjetivos semánticamente intensificados», es decir, adjetivos cuyo contenido semántico constituye la intensificación, a nivel paradigmático, de otro adjetivo (como excelente [bueno]; cfr. Albelda, 2004). La tabla 4 contiene una lista de «adjetivos semánticamente intensificados» en cada subcorpus, y su frecuencia normalizada por 1000 palabras.

\begin{tabular}{|l|l|l|l|}
\hline & \multicolumn{1}{|c|}{ ES_ES } & \multicolumn{1}{|c|}{ ES_INT_INT } \\
\hline & excelente & excelente & great \\
& precioso & precioso & excellent \\
hot \\
perfecto & estupendo & delicious \\
fantastic \\
Adjetivos semánticamente & estupendo & único & huge \\
intensificados & único & genial & amazing \\
& genial & ideal & encantador \\
& ideal & espectacular & superb \\
& inmejorable & \\
& inmejorable & & \\
\end{tabular}




\begin{tabular}{|l|c|c|c|}
\hline & $\begin{array}{l}\text { encantado } \\
\text { fenomenal }\end{array}$ & impresionante & \\
\hline Frecuencia/1 000 palabras & 13.47 & 8.47 & 6.78 \\
\hline
\end{tabular}

Tabla 4. Adjetivos semánticamente intensificados y su frecuencia

Las tablas 3 y 4 evidencian tendencias interesantes. Los tres subcorpus presentan una frecuencia parecida de adjetivos, ya que, en todos, de cada 1000 palabras, cerca de 50 son adjetivos. La principal diferencia atañe la variedad léxica de los adjetivos: como lo indicado por el type/token ratio, las reseñas de ES_ES muestran una tendencia mayor a repetir los mismos adjetivos, mientras que ES_INT e ING_INT usan una mayor variedad de estos. Además, aunque los tres subcorpus presentan una proporción significativa de adjetivos semánticamente intensificados; en ES_ES este número es un 60 \% mayor que en ES_INT y casi doble que en ING_INT. El dato es interesante por tres razones: primero, confirma la limitada variedad léxica de las reseñas españolas publicadas en plataformas nacionales; segundo, señala una tendencia significativamente más marcada a la intensificación léxica en ES_ES frente a las demás reseñas; tercero, reitera una convergencia entre las reseñas en español e inglés publicadas en plataformas internacionales, que comparten parecidos niveles de variedad léxica y un menor uso de adjetivos semánticamente intensificados. Estos elementos, junto con el uso de un léxico semánticamente vago ( $c f r$. tabla 2 ), vinculan el lenguaje usado en ES_ES al registro coloquial (Briz, 2001: 96).

\subsection{Atenuación e intensificación de los adjetivos con carga semántica negativa}

\subsubsection{Método}

El siguiente paso ha consistido en la selección de los adjetivos con carga semántica negativa en los tres listados de frecuencia y en el análisis de sus concordancias para identificar mecanismos de atenuación e intensificación. La decisión de centrar el análisis solo en los adjetivos con carga semántica negativa se debe a la voluntad de mantener separados casos de cortesía mitigadora (de los actos amenazadores de la imagen) y cortesía valorizante (de los actos de refuerzo de la imagen) ( $c f r$. Albelda, 2005), ya que implican mecanismos atenuantes e intensificadores distintos. En el caso de los adjetivos con carga semántica negativa, nos encontramos en presencia de actos amenazadores de la imagen (FTA) intrínsecamente descorteses; el uso de atenuantes correspondería, por lo tanto, a la voluntad de limitar el impacto negativo de los FTA, como en (8), mientras que los intensificadores demuestran la voluntad de quien escribe de enfatizar la opinión negativa expresada sobre los establecimientos, como en (9):

(8) El desayuno algo caro, pero correcto y abundante [ES_INT]

(9) Nunca en mi vida he visto un hotel tan malo [ES_ES] 
Es interesante notar la casi total correspondencia de adjetivos negativos en los tres corpus, lo que ha permitido una comparación directa. En ES_ES y ES_INT se han analizado los adjetivos caro, malo/peor, frío y pequeño, y en ING_INT los adjetivos small, cold, old, bad, expensive. Hay que señalar que frío, pequeño, small y old presentaban ejemplos de usos con connotación positiva o ambigua (por ejemplo, Un hotelito pequeñito muy acogedor, From old little streets facing the harbour till the dramatically masterpiece of Romanic art), que no se han tomado en consideración en el análisis.

La identificación de patrones léxicos indicativos de atenuación e intensificación se ha realizado inicialmente a partir de la extracción de clusters, es decir, agrupamientos de palabras frecuentes en el entorno derecho e izquierdo de cada adjetivo: de esta forma, se han obtenido clusters de entre 2 y 4 palabras de longitud, y se han seleccionado los que presentan patrones léxicos útiles para el análisis. La tabla 5 muestra los clusters considerados para el adjetivo caro/expensive (en la lista adjetivos y sustantivos aparecen lematizados). Para los demás adjetivos, los clusters considerados se podrán deducir de los ejemplos ilustrados en el análisis cualitativo.

\begin{tabular}{|l|l|l|}
\hline \multicolumn{1}{|c|}{ ES_ES } & \multicolumn{1}{|c|}{ ES_INT } & \multicolumn{1}{c|}{ ING_INT } \\
\hline caro, pero & caro para lo que & expensive though depending on \\
caro, pero está bien & caro y & expensive and of a \\
caro en mi opinión & caro, y & expensive at all \\
caro me parece & caro, pero & expensive at about full price \\
caro para el servicio & caro pero & expensive but \\
caro para estancias largas & caro para la categoría & expensive but if you \\
caro para la categoría & caro aunque & expensive car parking \\
caro para lo poco & caro para el & expensive considering \\
caro q son/es & caro para ser & expensive considering how \\
muy caro & caro porque & much \\
poco caro & caro que es & expensive disappointing \\
un poco caro & caro teniendo en & expensive food \\
es caro & caro, además & far too expensive \\
no es caro & caro a mi parecer & still far too expensive \\
y caro & algo caro & too expensive \\
aunque no es caro & demasiado caro & be expensive \\
bastante caro & muy caro & can be expensive \\
bastante caras & poco caro & quite expensive \\
COMENTAR LO CARO & un poco caro & but quite expensive \\
cómodo y nada caro & más caro & it wasn't expensive \\
económico pero tampoco & bastante caro & least it wasn't expensive \\
caro & es muy caro & are more expensive \\
es excesivamente caro & no es muy caro & isn't expensive \\
es nada caro & excesivamente caro & a bit expensive \\
es muy caro & no demasiado caro & and it is expensive \\
es carísimo & no excesivamente caro & just a bit expensive \\
muy muy caro & y caro & but would seem expensive \\
no es excesivamente caro & con lo caro &
\end{tabular}




\begin{tabular}{|c|c|c|}
\hline no es muy caro & $\begin{array}{l}\text { algo más caro } \\
\text { aunque algo caro } \\
\text { aunque bastante caro } \\
\text { aunque un poco cara }\end{array}$ & $\begin{array}{l}\text { city is very expensive } \\
\text { less expensive } \\
\text { most expensive } \\
\text { nice less expensive }\end{array}$ \\
\hline
\end{tabular}

Tabla 5. Clusters de los adjetivos caro/expensive analizados

El análisis de los clusters frecuentes utilizados en correspondencia de cada adjetivo ha permitido la identificación de una serie de mecanismos atenuantes e intensificadores, que se ilustran a continuación de acuerdo a las clasificaciones propuestas en Briz (2001) y Albelda (2007) para el español y Vázquez y Giner (2008) y (2009) para el inglés.

Para concluir el análisis, cada una de las ocurrencias de los adjetivos ha sido clasificada como atenuada, intensificada o neutra, lo que ha permitido (en el apartado 4.3.3) realizar un estudio cuantitativo de la distribución de estas estrategias en los tres corpus.

\subsubsection{Análisis cualitativo}

La atenuación en ES_ES y ES_INT atañe tanto a lo dicho como al decir. La atenuación del contenido proposicional utiliza mecanismos morfemáticos de modificación interna -diminutivos, como en (10)- y modificadores externos -cuantificadores, como en (11-12)-. Se considera modificador externo también el uso, muy frecuente, del adjetivo único, que delimita el alcance del adjetivo negativo (13):

(10) El desayuno es tipo buffet pequeñito [ES_INT].

(11) $[\ldots]$ aparcamiento un poco caro [ES_INT].

(12) un pueblo relativamente pequeño y muuuuuuuuuy tranquilo [ES_INT].

(13) La única pequeña pega es la situación [ES_INT].

En numerosos casos modificación interna y externa se combinan, con el uso de un diminutivo para modificar el cuantificador (14-15):

(14) [...] una televisión plana para mí un pelín pequeña [ES_INT].

(15) $[\ldots]$ consumir en la cafetería era un poquito caro [ES_INT].

Es muy frecuente el uso de la negación que acompaña formas intensificadas del adjetivo para negar una presuposición o expectativa implícita en el discurso (16-18), para cerrar una serie de adjetivos positivos (19) o después de una valoración positiva (20-21). En los casos como (19-21), es interesante notar la función de la conjunción y para marcar la transición de las evaluaciones positivas a la negación del adjetivo negativo: esa función se puede interpretar como adversativa y atenuadora (por ejemplo, un buen desayuno pero no excesivamente caro), o bien como aditiva e intensificadora de la serie de evaluaciones positivas (por ejemplo, un buen desayuno y encima no excesivamente caro): 
(16) Todo en su sitio, como si fuese un hotel de cadena, pero no tan caro [ES_INT].

(17) Y sin olvidar de que el hotel es un cuatro estrellas modernito y no demasiado caro [ES_INT].

(18) El desayuno buffet no es muy caro [ES_INT].

(19) Está bien situado, con buen desayuno y a un precio no excesivamente caro [ES_INT].

(20) El spa es un lujo la verdad, y no es nada caro [ES_ES].

(21) [...] están a unos metros y no son nada caros [ES_ES].

La negación de formas intensificadas del adjetivo puede ser seguida de un sintagma que restringe el alcance o la aplicabilidad de la calidad expresada por el adjetivo, atenuando su impacto (22-23):

(22) Agradable, cómodo y nada caro para la categoría que tiene [ES_ES].

(23) No es muy caro para el servicio que ofrece, prácticamente como el de un 4 estrellas [ES_ES].

La atenuación se realiza a menudo a través de proposiciones adversativas o concesivas, que yuxtaponen la propiedad negativa del adjetivo a una o más propiedades positivas con función compensativa (24-25). A menudo, el uso de estas proposiciones se asocia a otros mecanismos de modificación atenuadora (26-27):

(24) Ciudad pequeña pero encantadora [ES_INT].

(25) Es un hotel clasico en un edificio pequeño del centro de Valladolid, pero tiene todo lo que puedes necesitar [ES_INT].

(26) La casa tiene todo lo necesario y aunque pequeñita es muy coqueta [ES_INT].

(27) En definitiva, un buen hotel, nuevo, moderno y limpio, aunque un poco caro [ES_INT].

(28) El desayuno algo caro, pero correcto y abundante [ES_INT].

El potencial amenazador del adjetivo se limita en algunos casos a través de gerundios con funcional condicional, a través de los cuales quien escribe motiva el juicio expresado (29):

(29) El desayuno 4,50 € algo caro teniendo en cuenta que el café no es expreso [ES_INT].

La atenuación se manifiesta también a través de formas mitigadoras del decir, o de los actos ilocutivos, con el uso explícito del verbo performativo que enfatiza la subjetividad de la opinión expresada (30) y de verbos modales que expresan la modalidad epistémica de posibilidad (31). A menudo, la atenuación pragmática se combina con la atenuación semántica realizada por modificadores externos (32-33): 
(30) y te ofrecen el «set spa» (albornoz, gorro y sandalias) por 10 euros, caro me parece si ya has pagado por una habitación en dicho hotel [ES_ES].

(31) Si tienes la suerte de que te den vistas al Duero, como fue nuestro caso, no podrás pedir más. Y si no, tampoco debe ser malo, porque alrededor se extiende el verdor de los jardines y me da que hasta podría divisarse algo de la ciudad [ES_INT].

(32) [...] los dos coincidimos en que es un poco frío [ES_INT].

(33) La decoración, muy moderna, me resulta algo fría [ES_INT].

Muy numerosos también son los casos en que el uso del performativo expreso es modificado por una negación (34-38) o por el adverbio solo (39). En (34), la atenuación obtenida por la negación del verbo se combina con el efecto mitigador de la subordinada introducida por para en función de complemento de limitación. En (36-37), quien escribe responde a las expectativas impuestas por las convenciones discursivas del género (que prevé comentarios positivos y negativos), negándose a señalar las carencias del establecimiento. En (38), el autor usa la negación para contradecir las opiniones expresadas por otros viajeros, revelando la naturaleza interactiva de estos textos:

(34) Y no me pareció caro para ser un hotel de cinco estrellas [ES_INT].

(35) No digo que esté mala la comida solo pido variedad [ES_INT].

(36) Yo no destacaría nada malo del hotel [ES_INT].

(37) No puedo decir nada malo del servicio [ES_INT].

(38) Las habitaciones no me parecieron tan pequeñas como he leído en algún comentario [ES_ES].

(39) Solo me parecio un poco pequeño el armario de la ropa [ES_INT].

La fuerza ilocutiva de los actos lingüísticos se ve mitigada en ocasiones por las que Briz (2001: 152) modificaciones «al margen» (40-44), adverbios modalizadores (45-46) y el uso de formas impersonales (47):

(40) La verdad que aunque se haga un poco caro, lo merece [ES_INT].

(41) [...] minibar con precios un poco caros a mi parecer [ES_INT].

(42) Precios del parking; un poco caro en mi opinión [ES_ES].

(43) [...] el trato del personal en general es un poco frío [ES_ES].

(44) [...] una televisión plana para mí un pelín pequeña [ES_INT].

(45) [...] quizás algo pequeñas las habitaciones para los que busquen algo más que dormir [ES_ES].

(46) Lo peor tal vez las comidas [ES_ES].

(47) Los dos áticos son especialmente interesantes aunque pueden parecer pequeños [ES_INT].

Como han evidenciado los ejemplos hasta aquí ilustrados, la mitigación del potencial impacto negativo de los adjetivos analizados se realiza a través de una combinación de 
múltiples recursos atenuantes. La frase en (48) proporciona una demostración evidente de esta tendencia a la acumulación de fórmulas atenuadoras:

(48) Un pequeño defecto de la habitación quizá sea que la televisión no se veía demasiado bien, entiendo que quizá más por un problema de señal que otra cosa, pero sería algo en lo que desde el Parador deberían trabajar para solucionar, ya que en un par de ratillos que la encendimos era realmente desagradable [ES_INT].

Pasando ahora a considerar los mecanismos de intensificación, hay que señalar que también en este caso la modificación del contenido proposicional se realiza a través de recursos morfemáticos como los sufijos del superlativo, prefijos aumentativos $\mathrm{y}$, en el caso de mal, las formas superlativas peor y pésimo:

(49) El desayuno carísimo [ES_ES].

(50) [...] una habitación super pequeña con cama de matrimonio estándar [ES_INT].

(51) Las habitaciones de peor calidad y el servicio pésimo [ES_INT].

El valor semántico del adjetivo pequeño se ve reforzado cuando se asocia a sustantivos modificados por diminutivos como en (52):

(52) pero lo del champú y tal, te ponen sobrecitos pequeños, por lo que es recomendable llevarse el propio [ES_INT].

El grado de los adjetivos se modifica a menudo a través de modificadores simples (53-58), locuciones (59) o fraseologismos que expresan el superlativo (60); $c f r$. Albelda (2007):

(53) $[\ldots]$ el parking bastante caro [ES_ES].

(54) Parking muy caro [ES_ES].

(55) Mi opinión final acerca de este hostal es francamente mala [ES_INT].

(56) En invierno es frío literalmente [ES_INT].

(57) [...] hay detalles horrendos como el desayuno extremadamente caro con marcas hacendado o eroski [ES_INT].

(58) La comida (almuerzos o cenas) tiene escasa variedad para elegir y es excesivamente cara para la categoría del hotel [ES_INT].

(59) Ha sido con diferencia la peor experiencia de mi vida en un Hotel 5 Estrellas! [ES_INT].

(60) mas pequeña y justa imposible [ES_INT].

El efecto intensificador se obtiene a menudo con el uso de repeticiones (61-63) y enumeraciones que yuxtaponen adjetivos u otros elementos valorativos negativos seguidos (64-65), en algunos casos, de un comentario final que cierra la serie negativa (66). En 
ocasiones, el último elemento de la serie es introducido por un conector aditivo, que amplifica ulteriormente los efectos intensificadores de la acumulación (67-68):

(61) En primer lugar la comida, pésima es poco, mala, mala [ES_INT].

(62) Las habitaciones digamos que austeras y.. frías frías [ES_INT].

(63) El armario mиy, тиу pequeño, sin cajones [ES_ES].

(64) de nueva construcción, frio, vacío, poco acogedor, inacabado [ES_INT].

(65) Me asignaron habitación en la planta inferior: claustrofóbica, pequeña y casi sin luz [ES_ES].

(66) Pero la comida de lo peor, todo frito, con mal sabor, patatas rancias, mejor no recordar [ES_INT].

(67) El aparcamiento es muy escaso y además sale muy caro [ES_ES].

(68) Aparte de ser pequeña, se clavan los muelles [ES_INT].

Muy frecuente es el uso de los adjetivos analizados en construcciones sintácticas intensificadoras, como ilustrado en los siguientes ejemplos:

(69) [...] si quieres tomarte algo con lo caro que es y encima problemas con darte hielo [ES_INT].

(70) [...] por no contar lo seca, fría y mala que estaba toda la comida [ES_INT].

(71) Lo peor, lo mal que se regulaba la ducha y lo pequeña que era la bañera y la mampara [ES_ES].

Efectos de realce semántico-pragmático se obtienen también a través del uso del énfasis gráfico, que reproduce rasgos suprasegmentales enfáticos:

(72) Es verdad que es muuy pequeña para tantas habitaciones como tiene el hotel [ES_ES].

Antes de pasar a considerar los mecanismos utilizados en el subcorpus inglés, merece la pena subrayar que a menudo atenuación e intensificación conviven en una misma frase. En los ejemplos que siguen, las formas intensificadoras del superlativo se mitigan a través de elementos modales y la expresión del verbo performativo:

(73) Probablemente el peor parador nacional [ES_INT].

(74) El desayuno diría que es el peor de los aspectos del hotel [ES_INT].

(75) La unica pega $\mathrm{k}$ tiene es el spa como no tienen zapatillas ni gorros para los clientes ni tan solo para vender me parecio lo peor asi k le doy un 0 [ES_INT].

Los clusters del subcorpus inglés han evidenciado un sustancial solapamiento con los mecanismos atenuadores e intensificadores identificados en los subcorpus en español. La atenuación se realiza principalmente a través de premodificadores léxicos (76-77) y adversativas o concesivas (78-81): 
(76) Just a bit expensive at about $£ 70$ when there are a lot of deals out there [ING_INT].

(77) The place is a little bit small... [ING_INT].

(78) The car park rate is quite expensive but if you validate your ticket at reception on check out the rate drops to $£ 9.50$ per 24 hours [ING_INT].

(79) This is a small but perfectly formed park [ING_INT].

(80) Rooms, though a little small, were fine and clean [ING_INT].

(81) Despite being small the room had everything [ING_INT].

La negación es un recurso muy frecuente de atenuación, utilizado en combinación con adjetivos intensificados (82) y, en ocasiones, con rutinas lingüísticas o clichés (83-84):

(82) The bar is neither too big nor too small [ING_INT].

(83) [...] breakfast was not too bad at all [ING_INT].

(84) Not all bad [ING_INT].

Como ya se ha señalado en el caso del español, el uso de complementos de limitación contribuye a mitigar el potencial descortés del adjetivo:

(85) Not bad for the price [ING_INT].

(86) Room was small by some standards [ING_INT].

La atenuación pragmática se realiza a través de verbos modales que expresan la modalidad epistémica de posibilidad (87-89), adverbios modalizadores (88) y el uso de formas impersonales (89):

(87) Can be expensive... [ING_INT]

(88) The room was small and maybe a bit small for two people sharing [ING_INT].

(89) It's definitely a good hotel, but would seem expensive at full price [ING_INT].

La intensificación, por su parte, hace uso de la modificación léxica (bad/worse/worst, 90-92-95), de adverbios (91-96) y de la premodificación nominal (97-98):

(90) This sounds a really feeble complaint, but it is far worse than it sounds [ING_INT].

(91) The spa area was quite small [ING_INT].

(92) We've paid a lot more money \& got a lot worse [ING_INT].

(93) Parking in city is very expensive [ING_INT].

(94) my boyfriend complained he had a really bad nights sleep [ING_INT]

(95) This was by far the worst 4 star hotel I have stayed in [ING_INT].

(96) However what I found disappointing was the unusually small room [ING_INT].

(97) Others had problems with the water being stone cold! [ING_INT]

(98) On arrival the room was freezing cold [...] the heating was off so the room was ice cold again [ING_INT]. 
A nivel sintáctico recurren las consecutivas (99-101) y la enumeración (102-103), mientras que a nivel retórico se observa el uso constante de la hipérbole (104-105) y de enunciados fraseológicos (106-107):

(99) So bad its good [ING_INT].

(100) My room and the 2nd floor corridor smelled bad enough that I had to check to see if I was in a smoking area [ING_INT].

(101) One pool with jacuzzi attached but too cold to relax in [ING_INT].

(102) Cold, old and grim [ING_INT].

(103) Overpriced and small rooms [ING_INT].

(104) Dark, gloomy room with the worst bed in years! [ING_INT].

(105) I had the worst night sleep ever [ING_INT].

(106) Thinking it couldn't get any worse, we then went for our treatments [ING_INT].

(107) The lights were all wonky and to make things worse the noise from other guests was shocking [ING_INT].

Por último, como ya se había observado en los subcorpus en español, la intensificación se realiza con frecuencia a través de la modalidad exclamativa señalada a través del énfasis gráfico:

(108) Just want to say, what a small room! [ING_INT]

(109) COLD! [ING_INT]

\subsubsection{Análisis cuantitativo}

Como ya indicado en el apartado 4.3.1, la identificación de los mecanismos de atenuación e intensificación ha hecho posible catalogar cada una de las ocurrencias de los adjetivos analizados como atenuada, intensificada o neutra, permitiendo observar la distribución de estas categorías de uso en los tres corpus. En esta fase del estudio no se ha considerado el adjetivo inglés old, ya que no tiene un equivalente en los subcorpus en español y, por lo tanto, no se presta a la comparación cuantitativa. Tampoco se han computado los casos ambiguos en los que el adjetivo aparece modificado contemporáneamente por atenuantes e intensificadores. 


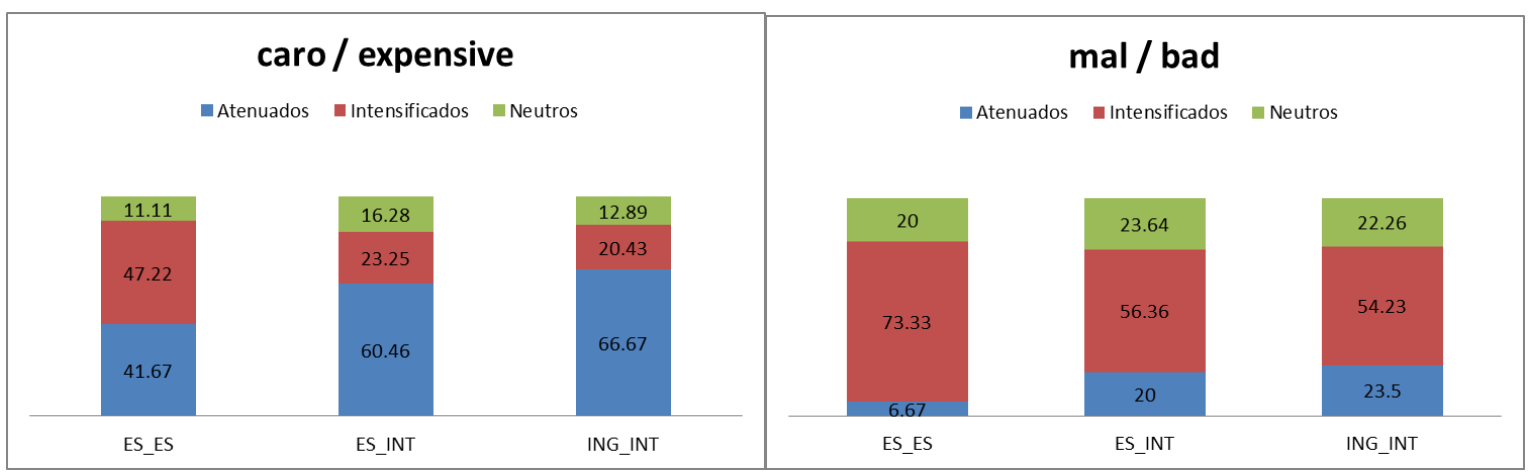

Figura 1. Distribución (en porcentajes) de usos atenuados, intensificados y neutros de los adjetivos carolexpensive y mal/bad en los tres subcorpus

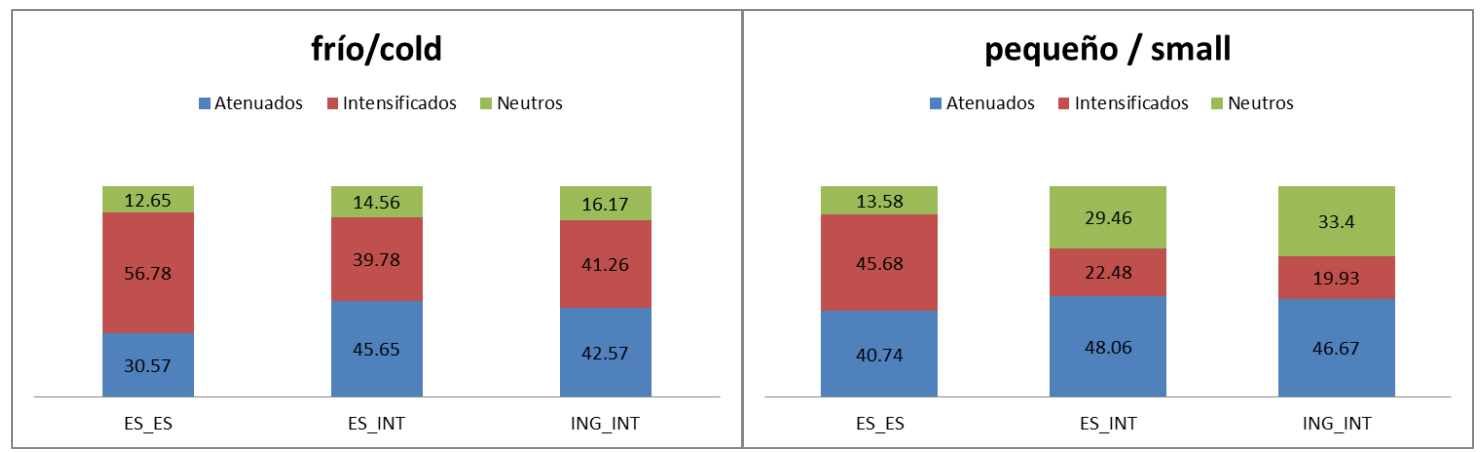

Figura 2. Distribución (en porcentajes) de usos atenuados, intensificados y neutros de los adjetivos frío/cold y pequeño/small en los tres subcorpus.

Las figuras 1 y 2 revelan que los adjetivos mal/bad presentan patrones de uso peculiares frente a los demás adjetivos, ya que se caracterizan por un marcado predominio de usos intensificados en los tres subcorpus: este hecho se puede explicar por la alta incidencia de las formas peor/worse y worst y por la indeterminación semántica del adjetivo, que lo asocia al registro informal y, por lo tanto, a las formas intensificadas.

Este equilibrio en la distribución de las categorías en los tres subcorpus no se observa para los restantes adjetivos, que muestran una clara tendencia al predominio de la intensificación en ES_ES y una preponderancia cuantitativa de la atenuación en ES_INT e ING_INT. Esta tendencia resulta particularmente evidente en el caso de carolexpensive y pequeño/small, para los que los usos intensificados en ES_ES tienen una incidencia de más del doble que en ES_INT e ING_INT.

Se confirman, de esta forma, los datos hasta ahora expuestos: las reseñas en español publicadas en plataformas nacionales presentan una tendencia más marcada al uso de la intensificación y, por lo tanto, un registro más cercano a lo coloquial; las reseñas en español publicadas en plataformas internacionales muestran una preferencia por formas atenuadas, 
que se pueden poner en relación con estrategias de cortesía mitigadora, típicas de situaciones caracterizadas por una mayor distancia social/funcional entre los interlocutores; típicas, en otras palabras, de contextos formales. Las analogías observadas en el uso de intensificación y atenuación en las reseñas en español y en inglés en plataformas internacionales parecen sugerir una convergencia interlingüística hacia un modelo globalizado, formalizado de acuerdo a un molde discursivo estable y compartido.

\section{CONCLUSIÓN}

El análisis basado en corpus propuesto en este trabajo ha comparado el uso de adjetivos en reseñas hoteleras en español y en inglés publicadas en plataformas nacionales e internacionales. Los resultados han evidenciado una mayor variedad léxica en el uso de adjetivos en las reseñas publicadas en web internacionales, mientras que en las nacionales se ha observado un más frecuente uso de adjetivos cuyo significado es de por sí intensificado (por ejemplo, fantástico, magnífico, perfecto, etc.). El análisis de los patrones léxicos recurrentes en el entorno inmediato de adjetivos semánticamente negativos ha demostrado una marcada tendencia a la intensificación en las reseñas nacionales, que se opone a una preferencia por formas atenuadas en las internacionales.

Estos datos han permitido colocar las reseñas en distintos puntos del continuum que une los dos polos de lo coloquial y lo formal, situando las reseñas publicadas en web nacionales en la llamada «constelación comunicativa coloquial», donde el uso de formas intensificadas promueve la visibilidad del autor y la expresión no mediada de sus actitudes y juicios, revelando además una supuesta proximidad (social, vivencial y funcional) entre quien escribe y quien lee; esta última se confirma por la limitada variedad léxica, que puede explicarse por el predominio del fin interpersonal de la comunicación. Las reseñas que aparecen en web internacionales, en cambio, presentan una menor asociación con los rasgos típicos de la comunicación informal, como demuestran la mayor variedad léxica (debida al fin transaccional de la interacción) y los recursos de cortesía mitigadora utilizados para reducir el potencial amenazador de la opinión negativa vehiculada por el adjetivo. Esta constante matización de lo dicho y la mitigación del compromiso de quien escribe con los actos lingüísticos realizados indican una mayor distancia entre emisor y receptor, típicas de contextos situacionales más formales.

A la luz de estos elementos, es posible reflexionar acerca de los efectos producidos por la comunicación entre pares en el mercado turístico globalizado. Un primer dato de interés lo proporcionan las convergencias evidenciadas por todos los parámetros analizados entre reseñas en español y en inglés publicadas en web internacionales: sorprende observar que las peculiaridades formales de cada idioma y los vínculos propios de sus respectivas tradiciones discursivas no influyen en los textos tanto como los rasgos situacionales en que estos nacen; los datos, de hecho, parecen sugerir que el uso de una plataforma global 
promueve la fidelidad al género internacional más que a la tradición discursiva de una lengua y cultura dadas.

Por otra parte, la apropiación de un género internacional por parte de una comunidad discursiva nacional se asocia, en el caso analizado, a un mayor nivel de formalidad de los textos, lo que apunta a la doble dinámica de popularización y especialización promovida por la comunicación en la red. En este estudio se ha confirmado la hipótesis avanzada al principio, evidenciando que la difusión de la comunicación entre pares en comunidades globales favorece la creación de moldes discursivos compartidos utilizados para fines transaccionales, donde se limitan los elementos subjetivos, expresivos y emotivos para dejar espacio a argumentaciones que revelan una voz matizada, equilibrada y autoritativa. En otras palabras, la comunicación entre pares, al obtener una función reconocida dentro de la comunidad discursiva de los viajeros, abandona los rasgos de la conversación informal para convertirse en una forma de comunicación especializada.

\section{REFERENCIAS BIBLIOGRÁFICAS}

AlBelda MARCO, Marta (2004): «La escalaridad en la intensificación», Interlingüística, 15, 105-114.

AlBELDA MARCO, Marta (2005): «Discordancia entre atenuación/cortesía e intensificación/descortesía en conversaciones coloquiales», en Blas Arroyo, José Luis, Mónica Velando Casanova y Manuela Casanova Avalos, coords., Discurso y Sociedad. Contribuciones al estudio de la lengua en contexto social, Castellón de la Plana, Servicio de publicaciones de la Universidad de Castellón, 581-590.

AlBELDA MARCO, Marta (2007): La intensificación como categoría pragmática: revisión y propuesta, Fráncfort del Meno, Peter Lang [en línea]: <http://dx.doi.org/10.1075/sic.6.2.08rui >.

ALBELDA MARCO, Marta (2008): «Influence of situational factors in the codification and interpretation of impoliteness», Pragmatics, 18(4), 751-773.

AlBELdA MARCO, Marta (2010): «¿Cómo se reconoce la atenuación? Una aproximación metodológica basada en el español peninsular hablado», en Orletti, Franca y Laura Mariottini, eds., (Des)cortesía en español. Espacios teóricos y metodológicos para su estudio, Roma, Università degli Studi Roma Tre y Programa EDICE, 47-70.

BRAVO, Diana (2008): «Situación de habla, recursos comunicativos y factores lingüísticos en la interpretación de objetivos de cortesía», en Briz, Antonio et alii, eds., Cortesía y conversación: de lo escrito a lo oral, Valencia, Universitat de València, 12-24.

BRIZ GóMEZ, Antonio (2001 [1998]): El español coloquial en la conversación. Esbozo de pragmagramática, Barcelona, Ariel.

BRIz GóMEZ, Antonio (2003): «La estrategia atenuadora en la conversación cotidiana española», en Bravo, Diana, ed., Actas del Primer Coloquio del programa EDICE. La 
perspectiva no etnocentrista de la cortesía: Identidad sociocultural de las comunidades hispanohablantes, Estocolmo, Universidad de Estocolmo, 17-46.

BRIZ GómEZ, Antonio (2004): «Cortesía verbal codificada y cortesía verbal interpretada en la conversación», en Bravo, Diana y Antonio Briz Gómez, eds., Pragmática sociocultural: estudios sobre el discurso de cortesía en español, Barcelona, Ariel, 67-93, [en línea]: 〈http://dx.doi.org/10.1075/sic.2.1.09ste>.

BRIZ GómEZ, Antonio (2010): «Lo coloquial y lo formal, el eje de la variedad lingüística», en Castañer Martín, Rosa María y Vicente Lagüéns Gracia, coords., De moneda nunca usada: Estudios dedicados a José M. ${ }^{a}$ Enguita Utrilla, Zaragoza, Instituto Fernando El Católico, CSIC, 125-133.

CALvi; Maria Vittoria (2011): «Las lenguas de especialidad», en Calvi, Maria Vittoria, Cristina Bordonaba Zabalza, Giovanna Mapelli y Javier Santos López, Las lenguas de especialidad en español, Roma, Carocci, 15-38.

Gretzel, Ulrike, Yoo, Kyung Hyan y Melanie PURIFOY (2007): «Online Travel Review Study: Role \& Impact of Online Travel Reviews», Laboratory for Intelligent Systems in Tourism, Texas A\&M University [en línea]: <http://www.tripadvisor.com/pdfs/OnlineTravelReviewReport.pdf $>$ [Consulta: 31/07/2014].

HAVERKATE, Henk (2004): «El análisis de la cortesía comunicativa, categorización pragmalingüística de la cultura española», en Bravo, Diana y Antonio Briz Gómez, eds., Pragmática sociocultural: estudios sobre el discurso de cortesía en español, Barcelona, Ariel, 55-65 [en línea]: 〈http://dx.doi.org/10.1075/sic.2.1.09ste>.

Hennig-ThuraU, Thorsten, GwINNER, Kevin P., WALSH, Gianfranco y Dwayne D. GrEmLER (2004): «Electronic Word-of-Mouth via Consumer-Opinion Platforms: What Motivates Consumers to Articulate Themselves on the Internet?», Journal of Interactive Marketing, 18, 1, 38-52 [en línea]: 〈http://dx.doi.org/10.1002/dir.10073〉.

Ho, Jason Y.C. y Melanie DEMPSEY (2010), «Viral marketing: Motivations to forward online content», Journal of Business Research, 63, 9-10, 1000-1006 [en línea]: $<$ http://dx.doi.org/10.1016/j.jbusres.2008.08.010>.

Hyland, Ken (1998): Hedging in Scientific Research Articles, Ámsterdam, John Benjamins [en línea]: 〈http://dx.doi.org/10.1075/pbns.54>.

MACKIEWICZ, Jo (2010): «Assertions of expertise in online product reviews», Journal of Business and Technical Communication, 24, 1, 3-28 [en línea]: <http://dx.doi.org/10.1177/1050651909346929>.

Mendiluce Cabrera, Gustavo y Ana Isabel Hernández Bartolomé (2005): «La matización asertiva en el artículo biomédico: una propuesta de clasificación para los estudios contrastivos inglés-español», Ibérica, 10, 63-90.

MEYER-HERMANN, Reinhard (1988): «Atenuación e intensificación», Anuario de Estudios Filológicos, 11, 275-279. 
Morales, Óscar Alberto, Daniel CASSAny Comas y Carolina GonZÁlez-PeÑa (2007): «La atenuación en artículos de revisión odontológicos en español: estudio exploratorio», Ibérica, 14, 33-57.

Oliver DEL Olmo, Sonia (2005-2006): «The Role of Passive Voice in Hedging Medical Discourse: a Corpus-based Study on English and Spanish Research Articles», LFE: Revista de lenguas para fines específicos, 11-12, 205-218.

TIAN, Youfei (2013): «Engagement in online hotel reviews: A comparative study», Discourse, Context \& Media, 2, 4, 184-191 [en línea]: <http://dx.doi.org/10.1016/j.dcm.2013.10.002>.

VÁZQUEZ, Ignacio y Diana GINER (2008): «Beyond Mood and Modality: Epistemic Modality Markers as Hedges in Research Articles. A Cross-Disciplinary Study», Revista Alicantina de Estudios Ingleses, 21, 171-190.

VÁzQuEZ, Ignacio y Diana GINER (2009): «Writing with Conviction: The Use of Boosters in Modelling Persuasion in Academic Discourses», Revista Alicantina de Estudios Ingleses, 22, 219-237.

Vermeulen, Ivar E. y Daphne SEegers (2009): «Tried and tested: the impact of online hotel reviews on consumer consideration», Tourism Management, 30, 1,123-127 [en línea]: 〈http://dx.doi.org/10.1016/j.tourman.2008.04.008>. 